\title{
Effects of wing size on magnetic levitation using coil induced field
}

\begin{abstract}
The paper discusses the effect of wing size on magnetic levitation using coil induced field for a proposed Levitator Guide (LG). The levitation consists of two main parts namely permanent magnet and current carrying copper coils. The variation of these parts may effects the produced levitated force. A few models have been analyzed with simulation. Results indicated that levitator guide model of wings with centered elbow give rise to the desired performance. Finally, a new type of levitator guide is proposed and fabricated.
\end{abstract}

Keyword: Magnetic fields; Magnetic levitation 